\title{
Teorías Implícitas y Prácticas de Enseñanza que Promueven la Inclusión Educativa en la Universidad. Instrumentos y Antecedentes para la Reflexión y Discusión
}

\author{
Constanza M. Herrera-Seda ${ }^{(1)}$, Claudia P. Pérez-Salas ${ }^{(2)}$ y Gerardo Echeita ${ }^{(3)}$ \\ (1) Departamento de Psicología, Oficina 16, Facultad de Ciencias Sociales, Universidad de Concepción. \\ Barrio Universitario s/n, Concepción, Región del Biobío, Chile (e-mail: constanzaherrer@udec.cl) \\ (2) Departamento de Psicología, Oficina 13, Facultad de Ciencias Sociales, Universidad de Concepción. \\ Barrio Universitario s/n, Concepción, Región del Biobío, Chile (e-mail: cperezs@udec.cl) \\ (3) Departamento Interfacultativo de Psicología Evolutiva y de la Educación, Despacho 108, Facultad de \\ Psicología, Universidad Autónoma de Madrid. C/ Iván P. Pavlov 6. Ciudad Universitaria de Canto Blanco, \\ Madrid, España (e-mail: gerardo.echeita@uam.es)
}

Recibido Ene. 25, 2016; Aceptado Mar. 11, 2016; Versión final May. 11, 2016, Publicado Oct. 2016

\section{Resumen}

Se presentan los resultados encontrados en la aplicación de dos instrumentos que exploran las teorías implícitas y las prácticas de enseñanza acerca de la inclusión educativa, en un grupo de docentes chilenos de pregrado. La diversificación del alumnado universitario en Chile ha traído consigo importantes desafíos para los docentes en términos de calidad y equidad de la educación, por lo que este estudio aporta ideas y reflexiones en este contexto. El análisis estadístico evidenció que los instrumentos presentaban adecuada validez y fiabilidad. Se encontró que las prácticas de enseñanza que favorecen la inclusión educativa se relacionaban significativamente con las concepciones sobre las capacidades de aprendizaje de los estudiantes, pero no con las concepciones sobre la responsabilidad de los docentes con el aprendizaje de los alumnos. Los instrumentos analizados mostraron ser útiles para la comprensión de las cogniciones, valores, y prácticas de los docentes universitarios.

\section{Implicit Theories and Teaching Practices that Promote Inclusive Education in the University. Instruments and Data for Reflection and Discussion}

\begin{abstract}
The results found from the application of two instruments that explore implicit theories and teaching practices about inclusive education in a group of undergraduate Chilean teachers are presented. Diversification of university students in Chile has generated major challenges to teachers, in terms of quality and equity in education and therefore this study contributes with ideas and reflections. The statistical analysis showed that the instruments employed in the study had adequate reliability and validity. It was also found that teaching practices that promote inclusive education are significantly related with conceptions about student's capabilities of learning, but they are not related with conceptions about teacher's responsibility with students learning. The instruments analyzed in this work showed to be useful for the comprehension of cognition, values, and practice of university teachers.
\end{abstract}




\section{INTRODUCCIÓN}

El propósito fundamental de este trabajo es aportar herramientas y evidencias empíricas que contribuyan a un proceso reflexivo de mejora de la calidad del sistema universitario, a partir del cambio en las prácticas y cogniciones que los docentes implementan para responder con equidad a la diversidad creciente del alumnado universitario chileno. En efecto, una serie de cambios sociales, políticos y económicos, que se sucedieron desde la década de 1980, se conjugaron para que se produjera un aumento exponencial de la cobertura y una gran diversificación de las características de los estudiantes (Sistema de Información de Educación Superior, SIES, 2015).

Las actuales características de los estudiantes han transformado las aulas universitarias en contextos heterogéneos. A la universidad asisten hoy en día un mayor número de mujeres, adultos que estudian por primera vez o que retornan al sistema educativo, extranjeros, alumnos/as que trabajan parte del tiempo 0 que poseen responsabilidad en el cuidado de familiares, estudiantes que provienen de familias pertenecientes a los quintiles más bajos de ingresos, que viven en condición de pobreza o que constituyen la primera generación en acceder a la educación superior (SIES, 2015).

La ampliación y diversificación de la matrícula universitaria no ha venido de la mano de políticas y estrategias educativas que garanticen que los estudiantes puedan concluir exitosamente su formación profesional, produciéndose importantes índices de abandono académico y de sobre duración de los estudios (SIES, 2015). Complementariamente, aunque la mayoría de los estudiantes experimenta vivencias positivas en la universidad existe un grupo relevante de alumnos/as en riesgo de exclusión por condiciones sociales, culturales o económicas, que cree enfrentarse a los desafíos académicos con escaso apoyo de sus docentes y pares (Abello et al., 2012). Este antecedente pone en evidencia que la calidad, en el caso de la enseñanza universitaria chilena, todavía es baja en la medida que no articula suficientemente los principios de excelencia y equidad de la formación (Marchesi y Martín, 2014).

\section{Inclusión educativa en la universidad}

En los últimos años los conceptos de equidad, atención a la diversidad e inclusión educativa han estado muy presentes en los debates sobre la calidad de la enseñanza. Mientras algunos autores tienden a considerarlos equivalentes, nosotros los vemos como aspectos complementarios. En este trabajo nos centramos en el desafío de la equidad desde el marco de referencia de la inclusión educativa. Este enfoque otorga énfasis al reconocimiento y la valoración de la diversidad de los estudiantes y sitúa el foco de las políticas educativas en la eliminación o reducción de las distintas barreras que dificultan la presencia todos los alumnos/as en un espacio formativo común, la participación de estos en el currículum y el logro de aprendizajes de calidad (UNESCO, 2008).

Entre las barreras existentes nos interesan las que se sitúan en el ámbito de los principios éticos, valores, concepciones y prácticas de los docentes, particularmente sobre las diferencias en el aprendizaje. En este sentido nos enfocamos en el estudio del modo como se comprende y responde a la diversidad, así como de la disposición de los profesionales hacia la mejora y la innovación, pues los entendemos como elementos clave en el proceso de transformación hacia prácticas y políticas más inclusivas (Booth y Ainscow, 2015).

Este ámbito de investigación es relevante por cuanto la literatura científica ha mostrado que en el contexto universitario las barreras para la inclusión, de distinto tipo y condición, tienden a superar los apoyos existentes (Gavira y Moriña, 2015). Ello pone de relieve la importancia de comprender la naturaleza de estas barreras y de proponer estrategias eficaces para su transformación en "facilitadores" de los procesos de inclusión. Las evidencias empíricas disponibles han indicado que las concepciones y prácticas docentes más inclusivas tienen efecto, no solo sobre el alumnado en condición de vulnerabilidad sino que, en la participación y el aprendizaje de todos los estudiantes (Shaw, 2009), lo que confiere a las investigaciones sobre inclusión educativa valor en la mejora de la enseñanza para todos.

\section{Teorías implícitas acerca de la inclusión educativa}

Hemos apuntado que nuestro interés se encuentra en el estudio de los principios éticos, valores y concepciones docentes acerca de la inclusión educativa. En este trabajo nos enfocamos en las concepciones docentes, las que pueden interpretarse desde distintos marcos de referencia teóricos. Nosotros hemos optado por abordarlas desde el marco conceptual de las teorías implícitas, según el cual las concepciones corresponden a creencias organizadas de manera relativamente coherente de acuerdo a principios implícitos (Pozo et al., 2006). 
En educación, dichas teorías versarían sobre la naturaleza del conocimiento y los procesos psicológicos que permiten la generación y transformación del conocimiento (Martín et al., 2014). En el caso de los docentes, estas cogniciones dan sentido a la enseñanza, influyendo a nivel cognitivo (por ejemplo, en el modo de interpretar la diversidad de los estudiantes al aprender) y guiando las decisiones y actuaciones en relación al currículum, la selección de los materiales, la implementación de estrategias de enseñanza, la evaluación, entre otros elementos (Martín et al., 2014).

La investigación en el contexto universitario ha permitido identificar diferentes concepciones. En cuanto a las teorías implícitas acerca del aprendizaje, García et al. (2014) corroboraron la propuesta de Pozo, et al. (2006), que distingue tres tipos de concepciones: directa, interpretativa y constructiva. Además, encontraron que en los docentes predominaban concepciones interpretativas del aprendizaje, las que convivían con concepciones constructivas y directas según la dimensión abordada. Lo anterior nos parece importante porque da cuenta de una multiplicidad de representaciones que coexisten en la mente de los docentes, que se activan de manera diferencial en distintos ámbitos de la enseñanza (Martín et al., 2014) y seguramente, ante estudiantes con distintas características y necesidades educativas.

Respecto a las concepciones de docentes universitarios acerca de la diversidad de sus estudiantes y su abordaje en el aula, se han descrito diferentes conceptualizaciones similares entre sí. Por ejemplo, la de Gordon et al. (2010) con docentes australianos o la de Amaro, et al. (2014) con docentes bolivianos. Estas han indicado que las concepciones de los docentes universitarios se organizan alrededor de dos dimensiones. En primer lugar, se encuentran las concepciones que abordan la noción de diversidad, las que se ubicarían en un continuo que va desde una visión homogénea, que no distingue diferencias entre los alumnos/as; pasando por una visión grupal, que reconoce distinciones derivadas de los contextos sociales; una visión individual, que identifica las diferencias personales al aprender; hasta otro extremo con una visión comprensiva que distingue tanto las diferencias grupales como individuales (Gordon et al., 2010).

En segundo lugar, se han descrito concepciones acerca del modo de responder a la diversidad, las que se ubican en un continuo en uno de cuyos extremos se ubica la posición de ignorar las diferencias de los alumnos/as, asignando a estos la responsabilidad única de adaptarse a la universidad; seguida por una posición intermedia de compensar las diferencias, apoyando a ciertos grupos o estudiantes identificados como distintos; y terminando, en el extremo opuesto, con la posición de aprovechar las diferencias como un recurso pedagógico que permite a todos ampliar su noción de diversidad (Gordon et al., 2010).

Aun cuando no se encuentre en el campo de la educación universitaria, la propuesta de Echeita, et al. (2013) nos puede iluminar acerca de una tercera dimensión que, complementariamente a las dos anteriores, ayuda a comprender las complejas concepciones docentes acerca de la diversidad. Esta dimensión da cuenta de las ideologías educativas que sostienen los valores y principios éticos que dan sentido a las acciones docentes, al tiempo que determinan la disposición de los docentes a la innovación y la mejora de sus propias prácticas y de las políticas de los centros en los que trabajan.

Las concepciones docentes pueden ser estudiadas tanto mediante técnicas de autorreporte, como a partir del registro de la actividad del sujeto (García et al., 2014). En esta investigación hemos optado por la utilización de un cuestionario de dilemas, que se sitúa en un plano intermedio entre estas técnicas. Este tipo de cuestionario permite y posibilita acceder a un número importante de sujetos. Los cuestionarios de dilemas han mostrado fiabilidad y aplicabilidad en diversos estudios anteriores en el campo educativo (ver por ejemplo, Pozo et al., 2006).

\section{Prácticas de enseñanza que promueven la inclusión educativa}

Las teorías implícitas se expresan, no tanto en el discurso declarado, sino sobre todo en las prácticas (Pozo et al., 2006). En este sentido, la investigación disponible nos ha permitido conocer gran variedad de prácticas que concretan y sostienen los valores y concepciones inclusivas, en las que el docente valora la diversidad en el aula y se compromete con el aprendizaje de todos los estudiantes (Booth y Ainscow, 2015).

De manera sintética, cabe resaltar algunos rasgos de las prácticas de enseñanza que promueven la inclusión educativa, como es proveer de un abanico de posibilidades para aprender, dentro del cual los alumnos/as tienen la oportunidad de elegir cómo, cuándo, dónde y con quién aprender (Florian y BlackHawkins, 2011); incrementar la agencia y confianza para aprender que poseen todos los estudiantes (Hart et al., 2007); favorecer la interacción y la cooperación entre compañeros/as para aprender cómo mejorar la convivencia (Barkley et al. 2007). Adicionalmente, las investigaciones con grupos de estudiantes en condición de vulnerabilidad han descrito que la enseñanza inclusiva promueve el desarrollo de ambientes de aula acogedores y seguros, donde las actividades y recursos llevan a los estudiantes a vincularse y a reflexionar acerca de la diversidad (Hockings et al., 2012). Las actividades de aprendizaje inclusivas 
consideran los valores de los estudiantes (Curtis et al., 2015), favorecen la participación y la actividad de todos los alumnos/as mediante el uso de materiales motivadores, la aplicación del conocimiento, el trabajo en grupos pequeños y el acceso a recursos tecnológicos (Gavira y Moriña, 2015).

Finalmente, las prácticas inclusivas en la universidad permiten a todos los estudiantes completar con éxito las actividades de aprendizaje, a partir del uso flexible del tiempo, la guía docente ante las dificultades de aprendizaje y el apoyo emocional para responder a las exigencias de las actividades académicas (Hughes et al., 2015). Desde este enfoque los docentes expresan de manera clara y sencilla los contenidos, las expectativas y los requisitos que se deben cumplir; enseñan los contenidos paso a paso, partiendo de los conocimientos previos; y solicitan a los estudiantes retroalimentación para mejorar su enseñanza (Devlin y O'Shea, 2012).

Desde nuestra perspectiva, los trabajos relacionados con el diseño universal del aprendizaje (DUA) y el diseño universal de la instrucción (DUI) articulan estos distintos hallazgos al proponer la diversificación de las formas de representación, expresión y compromiso del aprendizaje, tanto en el diseño general de la instrucción, como en los materiales, instalaciones y estrategias utilizadas (García y Cotrina, 2012; Ruiz et al., 2012). Estos diseños otorgan a los alumnos/as oportunidades de acceder a los recursos de aprendizaje a través de diversos medios y momentos, así como seleccionar las actividades de su interés, acrecentando su compromiso y rendimiento académico (Pisha y Coyne, 2001).

Las prácticas docentes han sido investigadas con distintas herramientas, estando su selección sujeta a los fines de cada estudio y a los recursos disponibles. Por un lado, están las que se sustentan en la percepción de un externo, siendo la observación la principal técnica de recolección. Estas observaciones permiten describir las prácticas de aula, pero no los aspectos que se dan fuera de esta, como la planificación. Por otro lado, están los instrumentos de autorreporte, que logran captar los distintos aspectos de la enseñanza y la subjetividad del docente, pero que pueden estar sujetos a sesgos en el reporte. Dentro de estos instrumentos, las escalas y cuestionarios tienden a ser los más frecuentes, por su utilidad para alcanzar a un gran número de sujetos logrando mayor representatividad (Creswell, 2013) y es la razón por lo que los hemos preferido en esta investigación.

En el presente trabajo nos focalizamos en el desarrollo de instrumentos que nos ayuden a comprender las cogniciones y conductas docentes que favorecen la inclusión educativa en la universidad. Lo anterior se justifica en la necesidad de contar con herramientas cuantitativas para la recolección de datos, hasta ahora inexistentes en el contexto nacional. Estos instrumentos permiten conducir procesos de evaluación que integren un elevado número de docentes y facilitan la estimación precisa de los avances que los profesores/as han alcanzado. Cabe indicar que el foco del uso de estos instrumentos no se debe orientar a la certificación o clasificación de los docentes, sino a su empleo como plataforma de reflexión colectiva y movilización de acciones que contribuyan hacia procesos educativos más inclusivos.

Los objetivos planteados en este trabajo son: (1) determinar las propiedades psicométricas de dos instrumentos que permiten conocer las cogniciones y prácticas acerca de la inclusión educativa en una muestra de docentes universitarios de la Región del Biobío, Chile; (2) describir las teorías implícitas y prácticas de enseñanza que promueven la inclusión educativa en docentes universitarios de la Región del Biobío, Chile; y (3) explorar la relación entre las teorías implícitas y las prácticas de enseñanza que promueven la inclusión educativa en docentes universitarios.

\section{METODOLOGÍA}

\section{Diseño de investigación}

La investigación que describimos fue desarrollada siguiendo un enfoque cuantitativo, bajo un diseño de encuesta y un alcance descriptivo de corte transversal. El diseño de encuesta permite identificar características, conductas, cogniciones, etc. de un grupo de sujetos y extrapolar los resultados a la población a la que pertenecen (Creswell, 2013). En este caso nos ha interesado indagar las características psicométricas de dos instrumentos que permiten recoger información acerca de la promoción de la inclusión educativa por parte de docentes universitarios, así como conocer los resultados evidenciados por un grupo de profesores/as de este nivel.

\section{Participantes}

En la investigación participaron 232 docentes, de 7 universidades de la Región del Biobío en Chile. En consideración a la clasificación de las disciplinas científicas y tecnológicas de la Comisión Nacional de Ciencia y Tecnología (CONICYT) los participantes se distribuyeron de la siguiente forma: 32,2\% realizaba 
clases en carreras de pregrado del área de Ciencias Sociales; $21,8 \%$ de Ciencias de la Ingeniería; 13,7\% de Ciencias Naturales y Exactas; 13,7\% de Ciencias Jurídicas, Económicas y Administrativas; 11,3\% de Ciencias de la Medicina; 5,0\% de Ciencias Silvoagropecuarias; y 2,3\% de Humanidades. En cuanto a las características de los participantes, 55,3\% eran hombres y 44,7\% mujeres; el 10,0\% tenía grado de licenciatura, $48,2 \%$ de magister y $41,8 \%$ de doctorado. En promedio, los docentes tenían 15 años de experiencia $(D E=11,7)$ y dedicaban semanalmente 21 horas $(D E=14,0)$ a actividades académicas.

Los participantes fueron seleccionados con un muestreo no probabilístico, intencionado y por conveniencia, siguiendo el criterio de disponibilidad de los docentes a participar del estudio. Como criterios de inclusión se consideró: tener al menos 1 año de experiencia como docente universitario; trabajar a jornada parcial o completa en la universidad de pertenencia; y haber realizado clases durante el año anterior a la recolección de los datos. El único criterio de exclusión fue que las clases impartidas durante el año anterior se realizaran en modalidad de trabajo individual (por ejemplo, guía de seminario, tesis o práctica profesional).

\section{Instrumentos}

Las teorías implícitas acerca de la inclusión educativa fueron medidas con el Cuestionario de Dilemas acerca de la Inclusión Educativa (CDIE), que corresponde a una versión adaptada del Cuestionario de Concepciones Docentes sobre Conductas Disruptivas en el Aula desarrollado por Urbina et al. (2011) para el contexto escolar. El cuestionario corresponde a un instrumento de autorreporte, que presenta situaciones conflictivas ocurridas cuando los docentes se enfrentan a los dilemas que supone la inclusión educativa.

El cuestionario de Urbina et al. (2011) aborda tres dimensiones: (1) concepciones acerca del papel que las diferencias entre los estudiantes tienen en el aprendizaje, es decir, las teorías implícitas de los docentes acerca de la enseñanza-aprendizaje; (2) concepciones acerca de la naturaleza del trabajo docente, esto es de sobre los principios y estrategias que guían la respuesta educativa para atender a la diversidad, y (3) concepciones acerca de la ideología educativa en la cual se enmarcan los valores de los profesores/as, sus posiciones acerca de la equidad en educación y la actitud que tienen hacia la mejora educativa. El cuestionario original se compone de 20 ítems, a los que los docentes deben responder seleccionado una de tres alternativas de respuesta que expresan distintas teorías implícitas. La versión original abordaba la inclusión educativa de escolares que presentan conductas disruptivas y fue validado con una muestra de 180 profesores/as chilenos presentando una adecuada fiabilidad ( $\alpha=0,75)$ (Urbina et al., 2011).

Para la adaptación de este cuestionario mantuvimos el sentido de las situaciones y de las alternativas de respuesta; y ampliamos las situaciones más allá de las surgidas ante conductas disruptivas (por ejemplo, los logros de aprendizaje, las relaciones entre pares, la participación, etc.) y contextualizamos los dilemas a la universidad considerando su cultura, estructuras, normas y actores. En la Tabla 1, presentamos un ejemplo de dilema en su versión original y adaptada.

Tabla 1. Comparación dilema 1 en versión original del cuestionario y versión adaptada para la presente investigación

Dilema original elaborado por Urbina et a. (2011) Un grupo de profesores comparte la sensación de que cada vez se hace más difícil desarrollar sus clases con tranquilidad, debido al alto número de conductas disruptivas a las que tienen que hacer frente. Plantean diferentes causas para explicar la aparición de este tipo de conductas:

1. En la enseñanza media no les enseñan a controlar su conducta en el aula y este es un problema que los alumnos/as traen a la universidad.

2. A los estudiantes que están constantemente pendientes de su celular o su computador no les interesa aprender ni se motivan por temas académicos.

3. Los estudiantes se aburren porque en la universidad no somos capaces de ofrecerles un modelo de aprendizaje motivador donde se sientan partícipes.
Dilema adaptado para CDIE

Un grupo de docentes comparte la sensación de que cada vez se hace más difícil desarrollar sus clases, debido a que los estudiantes están constantemente pendientes de su celular o mirando su computador. Plantean diferentes causas para explicar la aparición de este tipo de conductas:

1. Los padres han disminuido la autoridad y el control sobre sus hijos, y están tan abrumados por otros temas que delegan esa responsabilidad a la escuela.

2. A los alumnos que presentan conductas disruptivas no les interesa aprender ni se motivan por temas escolares.

3. Los alumnos se aburren porque en la escuela no somos capaces de ofrecerles un modelo de aprendizaje motivador donde se sientan partícipes.

Con la finalidad de determinar la utilidad de la versión adaptada para dar cuenta del constructo de interés, realizamos un Análisis de Frecuencia de las respuestas y un Análisis de Correspondencias Múltiples (ACM). Estos análisis nos llevaron a eliminar 8 de los 20 dilemas iniciales, que presentaban bajas medidas de discriminación $(M<0,10)$, lo que impedía diferenciar las elecciones docentes. Eliminar estos ítems nos permitió aumentar la precisión, pero significó descartar dos sub-dimensiones de la propuesta original, a saber, el modo en que se concibe la organización social del aula y la utilidad de las etiquetas en la atención a los estudiantes. 
La inexistencia de un instrumento que abordara las prácticas de enseñanza inclusivas, acorde a las características del medio chileno y a las condiciones del nivel universitario, nos condujo a elaborar una herramienta de medición. La Escala de Prácticas de Enseñanza Inclusiva (EPEI) fue desarrollada a partir de un estudio cualitativo anterior, con 301 estudiantes pertenecientes a 13 carreras de 3 universidades de la zona. Dicho estudio nos permitió identificar variadas estrategias de enseñanza que los alumnos/as creían favorecedoras de la inclusión educativa, las que se organizaron en dos dimensiones: enseñanza flexible en la planificación, instrucción y evaluación; y apoyo al aprendizaje basado el respeto, el acompañamiento y la participación de todos los actores.

En base a las estrategias identificadas elaboramos 43 ítems iniciales. Un grupo de 25 estudiantes de pregrado valoró la relevancia de estos para sus logros de aprendizaje, lo que nos permitió extraer 18 ítems. La validez de contenido de los ítems creados fue corroborada mediante el juicio de 5 expertos en el ámbito de la Psicología Educacional. Los jueces sugirieron algunas mejoras en la redacción de los ítems y propusieron 14 ítems adicionales para abarcar la complejidad del constructo, lo que dio origen a la versión de 32 ítems aplicada en este trabajo.

La EPEI explora la frecuencia con que los docentes implementan acciones que promueven la participación y el aprendizaje de todos los estudiantes, con especial atención en aquellos alumnos/as o grupos que se encuentran en riesgo de ser marginados o fracasar a lo largo del proceso educativo. La escala corresponde a un instrumento de autorreporte, en formato Likert, con 5 alternativas de respuesta que van desde "nunca o casi nunca" a "siempre o casi siempre". El análisis de los datos recogidos nos llevó a eliminar 6 ítems que presentaban un bajo coeficiente de homogeneidad (menor de 0,10), es decir, cuya relación con los restantes indicadores era limitada. Los 26 ítems restantes fueron analizados en este estudio, luego de corroborar su capacidad de discriminación de los mismos a partir de la comparación de grupos extremos mediante la prueba U de Mann-Whitney.

\section{Procedimiento}

La recolección de datos comenzó con el contacto a los decanos de las facultades o directores de las carreras participantes. Una vez autorizada la participación de los docentes, los contactamos vía correo electrónico, motivándoles a participar del estudio. A los docentes que expusieron su intención de estar en la investigación, les solicitamos la firma de un consentimiento informado, en el indicaban que conocían el estudio y participaban voluntariamente. Entregamos a los docentes los instrumentos en papel, les solicitamos completarlo en el plazo de 15 días y al finalizar el periodo realizamos la recogida. Luego de la aplicación, traspasamos los datos a formato digital y construimos una base de datos para su análisis mediante SPSS.

\section{RESULTADOS}

\section{Teorías implícitas acerca de la inclusión educativa}

A continuación presentamos los resultados de la aplicación del CDIE considerando tanto el análisis estructural como descriptivo de los mismos. En primer lugar exponemos el Análisis de Frecuencia de las respuestas a cada ítem siguiendo las dimensiones originales propuestas por Urbina, et al. (2011).

En la Tabla 2 exponemos las teorías implícitas de los docentes acerca de las diferencias en el proceso de enseñanza-aprendizaje. Encontramos que predomina una concepción del aprendizaje (ver ítem 2) basada en el enfoque constructivista, que pone énfasis en el análisis profundo y la elaboración colectiva de los saberes. Por su parte, las diferencias entre los estudiantes (ver ítems 1 y 3) son entendidas principalmente como emanadas de múltiples de factores (personales, sociales, históricos y culturales) susceptibles de ser modificados. En el ítem 3 observamos la convivencia de esta concepción con otra que comprende las diferencias surgidas exclusivamente de condiciones del contexto (calidad de la formación anterior, capital social, apoyo familiar, etc.), las cuales son imposibles de transformar mediante la formación.

En la Tabla 3 presentamos las teorías implícitas acerca de la naturaleza del trabajo docente. Observamos que algo más de la mitad de los docentes creen en una cultura profesional colaborativa y adhocrática (ver ítems 5 y 8), en cuyo marco las necesidades de los estudiantes son abordadas mediante la cooperación entre profesionales. En cuanto a las estrategias de respuesta educativa a las dificultades de los estudiantes encontramos 2 tendencias: en los ítems 4 y 6 predominan estrategias basadas en el diálogo, la colaboración y la diversificación de las actividades durante la clase para resolver las dificultades; en cambio en el ítem 7 destacaron estrategias de atención extra clase. Lo anterior nos lleva a pensar, que en general los docentes prefieren resolver las dificultades de los estudiantes dentro del aula y que las estrategias que utilizan dependen de la situación problemática particular a la que se enfrenten, pudiendo ser adecuado el apoyo externo al resolver problemas vinculados a la convivencia. 
Tabla 2. Porcentaje de respuestas docentes a dilemas acerca de la inclusión educativa en la dimensión papel de las diferencias en el aprendizaje

Subdimensión teorías del aprendizaje

Ítem 2. Algunos docentes están discutiendo sobre la función de los conocimientos previos de los estudiantes. ¿Qué hay que hacer con estos conocimientos previos en el aula?

\begin{tabular}{c|c|c}
\hline Directa & Interpretativa & Constructivista \\
15,8 & 22,7 & 61,5 \\
\hline
\end{tabular}

Subdimensión origen y modificación de la conducta

Ítem 1. Un grupo de docentes comparte la sensación de que cada vez se hace más difícil desarrollar sus clases, debido a que los estudiantes están constantemente pendientes de su celular o mirando su computador. Plantean diferentes causas para explicar la aparición de este tipo de conductas:

\begin{tabular}{c|c|c}
\hline $\begin{array}{c}\text { Individual y estático } \\
20,9\end{array}$ & Ambiental y externo & Multifactorial y sistémico \\
& 14,7 & 64,4
\end{tabular}

Ítem 3. En una reunión de docentes se está discutiendo el caso de un estudiante que desde hace varios meses acude al Centro de Apoyo al Estudiante de la universidad, al que fue derivado por problemas de rendimiento académico, y continúa teniendo malos resultados en todas las asignaturas. Para explicar lo sucedido se plantean distintos puntos de vista:

\begin{tabular}{c|c|c}
\hline Individual y estático & Ambiental y externo & Multifactorial y sistémico \\
16,2 & 44,5 & 39,3
\end{tabular}

Tabla 3. Porcentaje de respuestas docentes a dilemas acerca de la inclusión educativa en la dimensión naturaleza del trabajo del docente

\section{Subdimensión cultura profesional}

Ítem 5. El Centro de Estudiantes tiene interés en discutir con los docentes el reglamento académico de la carrera, específicamente en lo que refiere a las causales de baja académica. Hay diferentes opiniones entre el grupo de docentes:
Individualista y burocrática
13,5
Competitiva
26,3
Colaborativa y adhocrática 60,2

Ítem 8. En una facultad se ha creado un servicio de asesoría educativa formado por psicólogos, para apoyar a los estudiantes que presentan dificultades de motivación en las clases. En reunión los docentes discuten el tipo de apoyo que se espera de estos profesionales, surgiendo distintas miradas:
Individualista y burocrática 12,7 Competitiva 34,3
Colaborativa y adhocrática 53,0

Subdimensión estrategias de respuesta educativa

Ítem 4. En una reunión de carrera, un docente comenta que el otro día, mientras caminaba por la facultad, observó cómo algunos de sus estudiantes estaban discutiendo con garabatos y ofensas cuando intentaban ponerse de acuerdo sobre una actividad de la carrera. Aparecen entonces distintas posturas:
Omisión y marginación
10,9
Atención fuera de clase
5,7
Colaboración dentro de clase
83,4

Ítem 6. Un estudiante que estuvo recluido en la cárcel se incorpora a la carrera. Los docentes han observado que algunos de sus compañeros han comenzado a realizar comentarios discriminadores, interrumpiendo la clase y provocando tensiones con el resto del curso. Ante esta situación los docentes plantean distintas opciones:
Omisión y marginación 16,9
Atención fuera de clase 10,3
Colaboración dentro de clase 72,8

Ítem 7. En una reunión de carrera un docente comenta que tuvo que llamarle la atención frente a todo el curso a dos de sus estudiantes por discutir y tratarse ofensivamente durante la clase. Esta situación le preocupa puesto que frente a cualquier nimiedad estos estudiantes acostumbran enfrentarse. Aparecen entonces distintas posturas:

\begin{tabular}{c|c|c} 
Omisión y marginación & Atención fuera de clase & Colaboración dentro de clase \\
30,1 & 56,4 & 13,5
\end{tabular}

En la Tabla 4 presentamos las teorías implícitas en torno a los ideologías educativas de los docentes. Particularmente, en ideología y valores docentes encontramos 2 visiones: en el ítem 10 observamos el predominio de una concepción pluralista y comunitaria de la educación universitaria, que valora el acceso universal a la educación superior; en el ítem 12 en cambio la mayoría sostiene una visión liberal e individualista que destaca el valor del esfuerzo personal para superar los retos académicos.

En concordancia, observamos una concepción de equidad (ver ítem 11) basada en la igualdad de oportunidades, que promueve el acceso de todos los jóvenes a la formación, pero que entiende el aprendizaje como restringido a las capacidades de cada estudiante. Por último, encontramos que más de la mitad de los docentes mostraron una actitud optimista hacia la mejora educativa (ver ítem 9), lo que les motiva a implicarse en la reflexión y el desarrollo de estrategias para apoyar a los estudiantes.

Tras analizar la frecuencia de respuesta procedimos a identificar las dimensiones latentes de las teorías implícitas de los docentes. En concreto, conducimos un Análisis de Correspondencias Múltiples, lo que nos 
llevó a extraer 2 dimensiones estadística y conceptualmente relevantes. El detalle de las medidas de discriminación de los ítems cada dimensión se presenta en la Tabla 5.

Tabla 4. Porcentaje de respuestas docentes a dilemas acerca de la inclusión educativa en la dimensión ideología educativa

Subdimensión Ideologías y valores

Ítem 10. En una reunión de docentes se discuten las dificultades de aprendizaje que han presentado estudiantes que ingresaron con menores puntajes a la carrera. La discusión ha derivado hacia cuáles deberían ser los fines de la educación, emergiendo distintas perspectivas:
Liberal e individualista
Igualitarista y solidaria
19,9
Pluralista y comunitaria
11,7
68,4

Ítem 12. En una reunión los docentes están trabajando en la actualización del plan de estudios de la carrera y se han dado cuenta que los programas de las asignaturas tienen visiones muy distintas de la formación profesional. La discusión ha derivado hacia cuáles son los valores que deberían fundamentar la formación en la carrera, manifestándose tres visiones mayoritarias:

Liberal e individualista

50,9

Igualitarista y solidaria
28,3

28,3

Pluralista y comunitaria 20,9

Subdimensión equidad

Ítem 11. Un grupo de profesores está discutiendo acerca del crecimiento en la matrícula universitaria, y lo que implica la idea de que todos los estudiantes puedan llegar a la educación superior. Se han manifestado diferentes puntos de vista:

\begin{tabular}{c|c|c}
\hline Igualdad oportunidades & Igualdad oferta & Igualdad oportunidades y resultados \\
57,8 & 11,6 & 30,6
\end{tabular}

Subdimensión actitud hacia la mejora educativa

Ítem 9. Frente a la decisión de las autoridades de permitir la reincorporación de un estudiante de primer año que reprobó la mayor parte de las asignaturas, se convoca a una reunión de docentes para debatir acerca de esta decisión. Se plantean distintas opiniones en torno al tema:

\begin{tabular}{c|c|c} 
& \\
\hline Defensa statu quo & Conformista & Optimista \\
16,6 & 31,0 & 52,4 \\
\hline
\end{tabular}

Tabla 5. Medidas de discriminación ítems del Cuestionario de Dilemas acerca de la Inclusión Educativa (CDIE)

\begin{tabular}{lcccc}
\hline \multicolumn{1}{c}{ Dimensiones originales } & Dilemas & Dimensión 1 & $\begin{array}{c}\text { Dimensiones CDIE } \\
\text { Dimensión 2 }\end{array}$ & Media \\
\hline \multirow{2}{*}{$\begin{array}{l}\text { Papel de las diferencias en el } \\
\text { aprendizaje }\end{array}$} & Ítem 2 & 0,26 & 0,01 & 0,13 \\
& Ítem 1 & 0,35 & 0,05 & 0,20 \\
& Ítem 3 & 0,35 & 0,09 & 0,22 \\
\hline \multirow{3}{*}{ Naturaleza del trabajo docente } & Ítem 5 & 0,23 & 0,16 & 0,20 \\
& Ítem 8 & 0,24 & 0,11 & 0,17 \\
& Ítem 4 & 0,26 & 0,25 & 0,26 \\
& Ítem 6 & 0,28 & 0,11 & 0,20 \\
Ideologías educativas & Ítem 7 & 0,26 & 0,15 & 0,20 \\
& Ítem 10 & 0,24 & 0,26 & 0,25 \\
& Ítem 12 & 0,23 & 0,12 & 0,18 \\
& Ítem 11 & 0,06 & 0,32 & 0,19 \\
& Ítem 9 & 0,45 & 0,07 & 0,26 \\
\hline
\end{tabular}

La dimensión 1 explica el 66\% de varianza, presenta una elevada consistencia interna $(\alpha=0,75)$ y la distribución de las respuestas nos ha ha llevado a concluir que corresponde a las teorías implícitas acerca de la capacidad de aprendizaje de los estudiantes y su potencial de cambio. Esta dimensión describe el modo en que los docentes comprenden el origen de las habilidades de los estudiantes para aprender y el potencial de cambio de sus patrones de rendimiento y respuestas actuales (Hart et al., 2007).

Las concepciones docentes en esta dimensión se ubican en un continuo entre dos polos. A la base del polo de determinismo se encuentra la idea que el rendimiento académico se basa en diferencias en las capacidades subyacentes de aprendizaje que son inalterables (Hart et al., 2007). Esta perspectiva lleva a identificar solo a un pequeño grupo de estudiantes como diferentes, entender sus dificultades como déficits individuales y desarrollar acciones de apoyo fuera de aula orientadas a estos alumnos/as (Echeita et al. 2013). En el núcleo del polo de transformación se ubica la creencia que la capacidad de aprendizaje de los alumnos/as es modificable, ya que surge de la interacción entre condiciones externas, recursos internos y estados de ánimo, pudiendo incrementarse al ampliar las oportunidades de aprendizaje en el aula (Hart et 
al., 2007). Desde esta concepción las diferencias son identificadas en todos los estudiantes y valoradas como recursos; y los docentes organizan sistemas de apoyo mayoritariamente dentro del aula disponibles para todos los estudiantes (Echeita et al. 2013).

La dimensión 2 explica una menor proporción de la varianza (34,0\%) y presenta una menor consistencia interna $(\alpha=0,44)$. Nuestro análisis nos ha llevado considerar que corresponde al principio ético de responsabilidad docente, es decir, el sentido personal de obligación y compromiso con los logros de aprendizaje de los estudiantes.

Las respuestas de los profesores/as se ubicarían a lo largo de un continuo entre dos polos. El polo de baja responsabilidad se sustenta en la idea que las dificultades de aprendizaje deben ser resueltas por los mismos estudiantes o por otros actores distintos del docente (profesional, tutor, familiar, etc.), lo que lleva a comprender la enseñanza como una actividad orientada exclusivamente a proveer de oportunidades académicas. El polo de alta responsabilidad se basa en la convicción del docente acerca de su compromiso con la promoción del aprendizaje de sus estudiantes, llevándolo a asegurarse que estos aprovechen las oportunidades de aprendizaje y obtengan resultados académicos positivos. Desde esta concepción los profesores/as son responsables de la enseñanza, las relaciones con los estudiantes, la motivación y el desempeño que pueden promover en sus alumnos/as (Lauermann y Karabenick, 2013).

El análisis descriptivo de las dimensiones lo presentamos en la Tabla 6. En cuanto a las teorías implícitas acerca de la capacidad de aprendizaje observamos visiones diversas que tienden levemente hacia el polo de transformación $(M=-0,002$ y $D E=1,01)$. Respecto a la dimensión de responsabilidad observamos un rango aun más amplio de concepciones, que se ordenan hacia ambos polos $(M=-0,002$ y $D E=1,01)$.

Tabla 6. Teorías implícitas docentes acerca de la capacidad y responsabilidad en el aprendizaje

\begin{tabular}{lcccccc}
\hline \multicolumn{1}{c}{ Dimensiones } & $\begin{array}{c}\text { Número } \\
\text { participantes }\end{array}$ & Media & Mediana & $\begin{array}{c}\text { Desviación } \\
\text { estándar }\end{array}$ & Mínimo & Máximo \\
\hline Capacidad de aprendizaje & 232 & $-0,002$ & 0,17 & 1,01 & $-2,96$ & 1,48 \\
Responsabilidad por el aprendizaje & 232 & $-0,002$ & 0,01 & 1,01 & $-2,69$ & 3,15 \\
\hline
\end{tabular}

\section{Prácticas de enseñanza que promueven la inclusión educativa}

Los datos recopilados mediante la EPEI los sometimos a Análisis Factorial Exploratorio (AFE) para conocer la estructura latente a las prácticas de enseñanza inclusivas. Cabe indicar que corroboramos los supuestos para el uso de esta prueba estadística: los ítems de la escala se encontraban suficientemente vinculados para ser explicados por una variable mayor $(K M O=0,89)$ y se descartó la presencia de una matriz de identidad (Prueba de Esfericidad de Bartlet, $\chi^{2}=1428, p<0,001$ ).

El método de extracción de Máxima Verosimilitud y el método de rotación Varimax, nos llevó a extraer 3 factores, considerando los indicadores estadísticos y el sentido teórico de los datos. El modelo de 3 factores explica el 33,6\% de la varianza observada y resulta adecuado según el Test de Bondad de Ajuste $\left(\chi^{2}=234\right.$, $g l=187, p=0,01)$. Durante este análisis eliminamos 3 ítems de la escala que se alejaban conceptual y/o estadísticamente de los tres factores extraídos, estos son: realizo actividades fuera del aula que ayudan a los estudiantes a reforzar lo que hemos visto en clases; dedico tiempo de la clase a dialogar con los estudiantes acerca de los temas de la asignatura; y planifico evaluaciones que consideran las necesidades de los estudiantes.

El análisis de las cargas y los significados de los ítems nos llevo a definir los factores como se indica a continuación (ver Tabla 7). El primer factor contempla 8 ítems, explica el 26,8\% de la varianza, presenta una elevada fiabilidad $(\alpha=0,79)$ y lo hemos denominado enseñanza para un aprendizaje significativo, aludiendo a estrategias docentes que favorecen que los estudiantes alcancen un aprendizaje con significado y sentido. Estas prácticas permiten a todos los alumnos/as participar de las actividades, favorecen la construcción de significados y promueven intercambios con los aprendices que movilizan sus conocimientos previos y le llevan a asignar sentido a las actividades académicas (Coll, 1988).

El segundo factor contempla 7 ítems, explica 3,99\% de la varianza, presenta una elevada fiabilidad $(\alpha=$ $0,75)$ y creemos puede ser descrito como co-agencia, es decir, como las acciones desarrolladas por los docentes para involucrar a los estudiantes como socios activos y comprometidos con la mejora del proceso de aprendizaje (Hart et al., 2007). Los profesores/as comparten con los alumnos/as la responsabilidad por el aprendizaje y les ayudan a desarrollar habilidades para ser más eficaces para responder a este desafío. 
Tabla 7. Matriz de estructura de la Escala de Prácticas de Enseñanza Inclusiva

\begin{tabular}{|c|c|c|c|}
\hline Ítems & Factor 1 & Factor 2 & Factor 3 \\
\hline $\begin{array}{l}\text { 1. Digo a los estudiantes que deben escuchar las opiniones de sus } \\
\text { compañeros sin descalificarlas }\end{array}$ & 0,391 & 0,176 & 0,200 \\
\hline $\begin{array}{l}\text { 2. Ayudo a los estudiantes a resolver las actividades de la asignatura cuando } \\
\text { tienen dificultades }\end{array}$ & 0,140 & 0,085 & 0,536 \\
\hline $\begin{array}{l}\text { 3. Entrego ejemplos adicionales cuando algún estudiante tiene dificultades } \\
\text { para comprender los conceptos de la asignatura }\end{array}$ & 0,163 & 0,161 & 0,374 \\
\hline $\begin{array}{l}\text { 4. Respondo a las dudas de los estudiantes fuera de clase (atención en } \\
\text { oficina, e-mail, facebook, etc.) }\end{array}$ & $-0,058$ & 0,068 & 0,430 \\
\hline $\begin{array}{l}\text { 5. Sugiero a los estudiantes recursos complementarios para el aprendizaje } \\
\text { (lecturas, audiovisuales, digitales, etc.) }\end{array}$ & 0,144 & 0,542 & 0,254 \\
\hline $\begin{array}{l}\text { 6. Enseño a los estudiantes estrategias para que puedan aprender más en } \\
\text { las asignaturas }\end{array}$ & 0,199 & 0,733 & 0,158 \\
\hline $\begin{array}{l}\text { 7. Entrego a los estudiantes varias opciones de tareas o trabajos a realizar } \\
\text { entre las que pueden elegir según su interés }\end{array}$ & 0,210 & 0,327 & 0,069 \\
\hline $\begin{array}{l}\text { 8. Transmito a los estudiantes que me gusta lo que enseño, mostrándome } \\
\text { entusiasmado durante la clase }\end{array}$ & 0,242 & 0,158 & 0,451 \\
\hline $\begin{array}{l}\text { 9. Informo a los estudiantes de la utilidad que tendrán los aprendizajes de la } \\
\text { asignatura cuando sean profesionales }\end{array}$ & 0,441 & 0,258 & 0,488 \\
\hline 10. Felicito a los estudiantes por sus logros y/o avances en la asignatura & 0,449 & 0,280 & 0,389 \\
\hline $\begin{array}{l}\text { 11. Llamo a los estudiantes por su nombre } \\
\text { 12. Modifico la planificación del curso a lo largo del semestre agregando }\end{array}$ & 0,268 & 0,256 & 0,092 \\
\hline $\begin{array}{l}\text { contenidos, actividades o materiales que responden a los estilos de } \\
\text { aprendizaje de los estudiantes }\end{array}$ & 0,316 & 0,356 & 0,041 \\
\hline $\begin{array}{l}\text { 13. En la planificación del curso señalo distintos materiales que podrían } \\
\text { ayudar a los estudiantes a aprender (lecturas, videos, programas, sitios, etc.) }\end{array}$ & 0,212 & 0,432 & 0,183 \\
\hline $\begin{array}{l}\text { 14. Realizo distintos tipos de actividades durante el semestre, para que todos } \\
\text { los estudiantes tengan la oportunidad de desarrollar sus habilidades }\end{array}$ & 0,517 & 0,382 & 0,213 \\
\hline $\begin{array}{l}\text { 15. Recojo todas las opiniones de los estudiantes al analizar los temas de la } \\
\text { asignatura }\end{array}$ & 0,294 & 0,139 & 0,380 \\
\hline $\begin{array}{l}\text { 16. Realizo actividades que permiten a los estudiantes aplicar los contenidos } \\
\text { aprendidos en situaciones prácticas }\end{array}$ & 0,654 & 0,217 & 0,189 \\
\hline $\begin{array}{l}\text { 17. Voy relacionando los temas del curso con otros temas que los } \\
\text { estudiantes vieron antes, en esta u otra asignatura }\end{array}$ & 0,123 & 0,114 & 0,531 \\
\hline $\begin{array}{l}\text { 18. Realizo actividades para asegurar que los estudiantes han aprendido y } \\
\text { reforzar lo que sea necesario }\end{array}$ & 0,387 & 0,520 & 0,212 \\
\hline $\begin{array}{l}\text { 19. Realizo actividades en que los estudiantes deben cooperar con sus } \\
\text { compañeros }\end{array}$ & 0,596 & 0,341 & 0,203 \\
\hline $\begin{array}{l}\text { 20. En la revisión de las evaluaciones entrego a los estudiantes sugerencias } \\
\text { concretas para mejorar }\end{array}$ & 0,188 & 0,488 & 0,208 \\
\hline $\begin{array}{l}\text { 21. Realizo distintos tipos de evaluaciones a lo largo del semestre (orales, } \\
\text { escritas, trabajos, proyectos, etc.) }\end{array}$ & 0,576 & 0,157 & 0,218 \\
\hline $\begin{array}{l}\text { 22. Realizo evaluaciones que realmente recogen los elementos más } \\
\text { importantes de la asignatura }\end{array}$ & 0,164 & 0,137 & 0,437 \\
\hline 23. Realizo pruebas que incluyen distintos tipos de ítems & 0,518 & 0,275 & 0,043 \\
\hline
\end{tabular}

El tercer factor considera 8 ítems, explica en 2,84\% de la varianza, presenta una elevada fiabilidad ( $\alpha=$ $0,73)$ y lo hemos reconocido como apoyo para atender a la diversidad, incluyendo una serie de acciones orientadas a responder a la diversidad de los estudiantes; contribuir a superar las barreras presentes en la cultura, las políticas o las prácticas educativas; y fomentar el acceso, la participación, el aprendizaje y el desarrollo de todos los alumnos (Booth y Ainscow, 2015).

En cuanto a los resultados en estos 3 factores (ver Tabla 8) encontramos que los docentes desarrollaban habitualmente acciones que promovían la inclusión educativa. En concreto, las acciones realizadas con mayor frecuencia correspondían a acciones de apoyo para atender a la diversidad $(M=35,5 ; D E=3,24)$, seguidas por el uso de estrategias de enseñanza orientadas al logro de un aprendizaje significativo $(M=$ $31,9 ; D E=5,31)$ y finalmente el empleo de acciones que promueven la co-agencia $(M=25,5 ; D E=4,59)$.

Tabla 8. Prácticas de enseñanza que promueven la inclusión educativa desarrolladas por los docentes

\begin{tabular}{lcccccc}
\hline \multicolumn{1}{c}{ Factores } & Número & & \multicolumn{2}{c}{ Desviación } \\
& participantes & Media & Mediana & estándar & Mínimo & Máximo \\
\hline Enseñanza para un aprendizaje significativo & 221 & 31,9 & 33,0 & 5,31 & 11,0 & 40,0 \\
Co-agencia & 225 & 25,5 & 26,0 & 4,59 & 11,0 & 34,0 \\
Apoyo para atender a la diversidad & 220 & 35,5 & 36,0 & 3,24 & 20,0 & 40,0 \\
\hline
\end{tabular}




\section{Vinculación entre las variables de interés}

Con la finalidad de explorar la relación existente entre las variables investigadas utilizamos la Prueba $r$ de Spearman. Como exponemos en la Tabla 9, no encontramos una relación significativa entre las teorías implícitas de los docentes, es decir, entre las concepciones acerca de la capacidad de aprendizaje de los alumnos/as y el sentido de responsabilidad con el aprendizaje de los estudiantes que tienen los docentes.

Tabla 9. Correlación entre teorías implícitas y prácticas de enseñanza que fomentan la inclusión educativa

\begin{tabular}{|c|c|c|c|c|}
\hline & $\begin{array}{l}\text { Responsabilidad } \\
\text { aprendizaje }\end{array}$ & $\begin{array}{c}\text { Enseñanza para } \\
\text { aprendizaje significativo }\end{array}$ & Co-agencia & $\begin{array}{c}\text { Apoyo para atender } \\
\text { diversidad }\end{array}$ \\
\hline \multicolumn{5}{|l|}{ Teorías implícitas } \\
\hline $\begin{array}{l}\text { - Capacidad } \\
\text { aprendizaje }\end{array}$ & $\begin{array}{c}0,020 \\
(p=0.77)\end{array}$ & $\begin{array}{c}0,34 \\
(p<0,001)\end{array}$ & $\begin{array}{c}0,25 \\
(p<0,001)\end{array}$ & $\begin{array}{c}0,21 \\
(p=0,002)\end{array}$ \\
\hline $\begin{array}{l}\text { - Responsabilidad } \\
\text { aprendizaje }\end{array}$ & & $\begin{array}{c}0,088 \\
(p=0,19)\end{array}$ & $\begin{array}{c}0,077 \\
(p=0,082)\end{array}$ & $\begin{array}{c}0,040 \\
(p=0,56)\end{array}$ \\
\hline \multicolumn{5}{|l|}{ Prácticas de enseñanza } \\
\hline $\begin{array}{l}\text { - Enseñanza para } \\
\text { aprendizaje significativo }\end{array}$ & & & $\begin{array}{c}0,66 \\
(p<0,001)\end{array}$ & $\begin{array}{c}0,57 \\
(p<0,001)\end{array}$ \\
\hline - Co-agencia & & & & $\begin{array}{c}0,51 \\
(p<0,001)\end{array}$ \\
\hline $\begin{array}{l}\text { - Apoyo para atender } \\
\text { diversidad }\end{array}$ & & & & \\
\hline
\end{tabular}

En cuanto a las teorías implícitas acerca de la capacidad de aprendizaje de los estudiantes encontramos que esta dimensión se relaciona de manera directa, significativa, aunque con baja intensidad, con los tres factores de prácticas de enseñanza que promueven la inclusión educativa, es decir, con las estrategias de enseñanza que promueven un aprendizaje significativo $(r=0,34 ; p<0,001)$, con la co-agencia $(r=0,25 ; p<$ $0,001)$, y con el apoyo para atender a la diversidad $(r=0,21 ; p=0,002)$. Lo anterior indica, que en la medida que las concepciones de los docentes se acercan más hacia el polo de transformación, los profesores/as reportan con más frecuencia estrategias de enseñanza que promueven la inclusión educativa. En cambio, vimos que el principio ético de responsabilidad no se encuentra vinculado de manera significativa con ninguno de los tipos de prácticas de enseñanza inclusiva.

Respecto a la vinculación entre las prácticas de enseñanza encontramos relaciones positivas, moderadas y significativas entre los tres factores evaluados. Al respecto, verificamos que cuando los docentes reportan emplear con más frecuencia estrategias de enseñanza orientadas al logro de un aprendizaje significativo, también indican desarrollar con mayor regularidad prácticas que favorecen la co-agencia $(r=0,66 ; p<$ $0,001)$ y practicas de apoyo para atender a la diversidad de los estudiantes $(r=0,57 ; p<0,001)$. Asimismo, un mayor empleo de estrategias que favorecen la co-agencia se relaciona significativamente con un mayor uso de estrategias orientadas a apoyar a los estudiantes atendiendo a su diversidad $(r=0,51 ; p<0,001)$.

\section{DISCUSIÓN}

A continuación analizamos los principales resultados encontrados en la investigación. En primer lugar la adaptación del cuestionario de dilemas, que realizamos para su ajuste desde el nivel escolar al superior, mostró ser adecuada para distinguir entre las concepciones de los docentes universitarios. La estructura de dos dimensiones del CDIE mostró se coherente con conceptualizaciones anteriores en torno al tema.

La primera dimensión encontrada aborda las concepciones de los profesores/as acerca del origen y la posibilidad de cambio la capacidad de aprendizaje de los estudiantes. Las respuestas docentes a esta dimensión se ubican en un continuo que ya había sido descrito por Hart et al. (2007) y Urbina et al. (2011) tras analizar las perspectivas de profesores del nivel escolar. Dicho continuo va desde un polo determinista, que concibe las capacidades como atributos fijos, a otro transformable, que las considera modificables a partir del proceso de enseñanza-aprendizaje.

Asimismo, la variedad de concepciones docentes en esta dimensión se condicen con los trabajos del campo universitario que indicamos en los antecedentes de este trabajo. El extremo del polo determinista de las capacidades de los estudiantes se corresponde con la noción de homogeneidad propuesta por Gordon et al. (2010), mientras que el extremo del polo transformable de las habilidades de los alumnos es un equivalente a la visión comprensiva propuestas por estos mismos autores. 
En esta dimensión, encontramos que las respuestas de los profesores/as eran variadas, pero que tienden a ubicarse hacia el polo de capacidad transformable. Lo anterior resulta un hallazgo de interés si se compara con estudios anteriores en torno al tema. A modo de ejemplo, Amaro et al. (2014) encontraron que las perspectivas de los docentes presentan una fuerte tendencia a situarse en un paradigma del déficit, a diferencia con lo ocurrido con los docentes chilenos.

Las diferencias identificadas podrían dar cuenta de distinciones efectivas entre los docentes, que emanan de los contextos de socialización de distintos grupos. Sin embargo, debemos reconocer que también podrían vincularse a la fuente de reporte de los datos, ya que mientras en el trabajo de Amaro et al. (2014) procede de un sujeto externo, en el caso de esta investigación hemos optado por conocer la percepción de los mismos docentes. Lo que pone de manifiesto la dificultad que implica acercarse a estas cogniciones docentes y la importancia de contar con múltiples fuentes de información.

En segundo lugar, identificamos el principio ético de responsabilidad con el aprendizaje de los estudiantes, que refiere al sentido de compromiso de los docentes con los logros de aprendizaje alcanzados por los alumnos/as. Las respuestas en esta dimensión se situaron en un continuo que iba desde un polo de baja de responsabilidad a otro de alta responsabilidad. Al respecto, encontramos que las concepciones docentes sobre responsabilidad eran altamente diversas y se ubicaban a lo largo de todo el continuo, sin una tendencia específica hacia uno de los polos.

El análisis de la relación entre estas dos dimensiones no permitió comprobar una relación significativa, lo que consideramos debe ser revisado nuevamente en futuros estudios. La investigación en torno al tema ha mostrado que las creencias docentes acerca de las capacidades de los estudiantes se encuentran vinculadas con su sentido de responsabilidad. Los docentes que consideran que el aprendizaje depende de características personales, que no pueden ser modificadas, se sienten menos comprometidos con el aprendizaje de sus alumnos/as y viceversa (Diamond et al., 2004).

Más allá de los resultados, nos parece plausible la correlación descrita en la literatura y creemos que los resultados podrían deberse a limitaciones de esta investigación. Por una parte, la menor proporción de varianza explicada por la dimensión de responsabilidad puede dar cuenta de una limitada sensibilidad del cuestionario para explorar el constructo de interés, por lo que en futuros estudios este instrumento requiere ser revisado para garantizar que los dilemas lo aborden suficientemente. Asimismo, la técnica analítica utilizada tiende a dificultar la comprensión de la interacción entre las dimensiones, ya que el ACM las ubica de manera ortogonal impidiendo tener claridad de la relación entre las concepciones identificadas.

Complementariamente, la ausencia de relación entre la responsabilidad de los docentes con el aprendizaje de los estudiantes y sus prácticas de enseñanza, nos conduce a cuestionarnos el carácter de esta concepción. Desde nuestra perspectiva, la responsabilidad percibida por el docente puede no corresponder al universo de las cogniciones, sino al de los principios éticos. Aunque sostenido en términos de ideario, este compromiso podría no lograr transferirse a la práctica cotidiana, ya que una serie de barreras del entorno universitario dificultan su materialización (tales como la falta de dominio de estrategias de enseñanza, la baja autoeficacia para atender a la diversidad en el aula o el bajo sentido de pertenencia a la institución universitaria).

Más allá de la falta de claridad acerca de esta dimensión seguimos sosteniendo la relevancia de abordar las cogniciones latentes de los docentes y el modo en que estas se organizan para constituir verdaderas teorías implícitas que dan sentido a la enseñanza y la relación de los profesores/as con sus estudiantes. Los dilemas que hemos propuesto contribuyen a tener una aproximación general acerca de las diversas concepciones de los educadores y son un medio a través del cual cada persona puede reflexionar sobre sus ideas y cuestionarlas en miras de lograr un cambio conceptual. Dicha reflexión en todo caso, podrá verse facilitada si en las instituciones se favorecen espacios de trabajo colaborativo, en que los docentes puedan analizar sus concepciones y prácticas con otros actores y/o "amigos críticos" (Booth y Ainscow, 2015).

Por otro lado, en este trabajo nos abocamos a analizar los resultados de una escala que explora las prácticas de enseñanza que utilizan los docentes para favorecer la inclusión en las aulas universitarias. El proceso de construcción nos llevó a concluir que existe una gran diversidad de prácticas que tienen el potencial de mejorar el aprendizaje y la participación de todos los alumnos/as. En un vasto universo de características de estudiantes y docentes, así como de contextos educativos, existe también un número diverso de acciones que podemos emprender para generar espacios de aula inclusivos y su estudio mediante una escala predefinida supondrá en todos los casos un acto de reducción artificial del que somos conscientes. 
Sin embargo, creemos que hemos logrado identificar una serie de acciones a partir de las cuales podemos acercarnos al constructo de interés y que pueden ser relevantes para conducir procesos iniciales de cambio en la enseñanza universitaria hacia una perspectiva y acción más inclusiva. Tal como indicamos al inicio de este trabajo la inclusión educativa constituye un proceso de transformación de las instituciones educativas y el análisis de los modos en que los docentes afrontan la actividad de aula constituye una importante fuente de información, que tiene el potencial de movilizar una mejora inclusiva a nivel institucional si se orienta en base valores inclusivos y lograr influir en los distintos niveles de la organización (Booth y Ainscow, 2015), de allí la importancia de contar con datos precisos sobre los que sea posible reflexionar y establecer acciones de mejora.

El análisis de los datos recogidos evidenció que las prácticas de enseñanza inclusiva pueden sintetizarse en tres factores: estrategias que promueven aprendizajes significativos, estrategias que fomentan la co-agencia y estrategias de apoyo para atender a la diversidad. Los docentes participantes, aunque presentaron resultados diversos, reportaron con elevada frecuencia prácticas de enseñanza que promueven la inclusión educativa, especialmente en lo que refiere al apoyo de los estudiantes para responder a sus necesidades y características diversas; y el desarrollo de actividades que promuevan un aprendizaje con significado y sentido. Estos resultados deben ser considerados con mesura, ya que variadas investigaciones han evidenciado que las prácticas reportadas por los docentes pueden no estar relacionadas con las prácticas realizadas efectivamente en aula (ver por ejemplo, Mansour, 2013).

Adicionalmente, mirar la elevada frecuencia con que los docentes reportan prácticas de enseñanza inclusiva, a la luz de las investigaciones que han reportado la insatisfacción de los estudiantes por ambientes de aula donde tienden a ser marginados nos lleva a considerar la necesidad de elaborar en el futuro una escala que aborde las prácticas docentes que constituyen barreras para el aprendizaje y la participación. Al respecto los trabajos en el nivel universitario han evidenciado diversas prácticas que favorecen la marginación o el fracaso académico. Por ejemplo, se ha observado que en ocasiones los docentes se muestran escasamente accesibles para los alumnos/as, emplean estrategias de enseñanza o evaluación que generan elevado estrés e incluso tienden a estigmatizar o burlarse de los alumnos que pertenecen a grupos sociales que poseen escasa representatividad en la universidad (Curtis et al., 2015).

De este modo, avanzar en el logro de climas de aula que acojan a los alumnos/as y promuevan su aprendizaje implica tanto incrementar las prácticas que favorecen la inclusión educativa, como reducir aquellas que la dificultan. En consecuencia, el estudio de este proceso requiere de instrumentos que permitan abordar la relación dialéctica entre inclusión y exclusión que ha sido descrita con anterioridad (Echeita et al. 2013) y creemos que este elemento constituye una de las principales líneas de investigación que pueden abordarse en futuros trabajos.

Respecto a cada uno de los factores identificados, nos parece relevante que el aspecto en que los docentes han alcanzado un mayor desarrollo corresponde al apoyo que estos entregan para responder a la diversidad de los estudiantes. Complementariamente, en la investigación encontramos que este tipo apoyo se relaciona manera significativa con las teorías implícitas acerca de la capacidad de aprendizaje de los estudiantes y su posibilidad de modificarse a lo largo de la formación universitaria. Este hallazgo es coherente con las investigaciones que han mostrado los efectos positivos de integrar la acción tutorial como estrategia docente para apoyar a los distintos alumnos/as (Amaro et al., 2014; Mas y Olmos, 2012) y desde nuestro punto de vista representa uno de los elementos centrales de la práctica docente para favorecer la inclusión educativa, en tanto facilita que los docentes confíen en que sus estudiantes podrán alcanzar los aprendizajes esperados, lo que ha sido destacado previamente por Hart et al. (2007).

Las estrategias de enseñanza que promueven aprendizajes significativos mostraron ser igualmente una práctica docente implementada con regularidad, aunque con menor frecuencia que el uso de las estrategias de apoyo. Al respecto, nos interesa recalcar que al igual que en investigaciones anteriores hemos observado que promover la inclusión educativa desde dentro del aula requiere del desarrollo de prácticas que fomenten un aprendizaje cooperativo (García y Cotrina, 2012; Mas y Olmos, 2012). En este sentido la dimensión de prácticas de enseñanza que favorecen aprendizaje con significado y sentido considera a partir de diversos indicadores la construcción colectiva del conocimiento, que se da a partir de la cooperación entre estudiantes y entre alumnos/as y docentes.

En cuanto a las estrategias que favorecer la co-agencia, observamos que estas prácticas son las que los docentes reportan con menos frecuencia. Lo anterior es relevante, ya que el logro de la autonomía en el aprendizaje ha sido identificado como un elemento importante en la formación universitaria (Hockings, et al. 2012) y podría vincularse con los hallazgos de Amaro et al. (2004) que han evidenciado el escaso desarrollo de prácticas de gestión del aula para el logro de un aprendizaje activo. Creemos que este resultado se puede relacionar, entre otras variables, con que un importante número de docentes carecen de formación 
especializada para la enseñanza en la universidad y que gran parte de las instancias de habilitación existentes se basan en formulaciones y hallazgos provenientes de la educación escolar, lo que refuerza la relevancia de construir herramientas e investigar la inclusión educativa en la universidad, con la finalidad de identificar elementos propios si los hubiera.

A partir del análisis de las relaciones entre las variables estudiadas, concluimos la existencia de una relación significativa entre los distintos tipos de prácticas de enseñanza que promueven la inclusión educativa. Lo anterior evidencia la importancia de establecer un conjunto organizado de prácticas, que desde sus distintas aristas contribuyan a que los alumnos/as puedan aprender y progresar en la formación universitaria. Al respecto, Hockings, et al. (2012), a partir de su experiencia en el desarrollo de iniciativas orientadas a promover la inclusión en las aulas universitarias, llaman la atención acerca de la importancia de comprender la diversidad de los estudiantes desde una visión multifacética, que reconoce la gran gama de características, necesidades e intereses de los alumnos. En consecuencia los autores concluyen la importancia de desarrollar una serie de estrategias vinculadas entre sí, que contribuyan a generar ambientes de aula seguros, donde el aprendizaje surge de la colaboración.

Asimismo, el estudio de la relación entre teorías implícitas y prácticas de enseñanza que promueven la inclusión educativa nos llevó a corroborar esta vinculación al menos en lo que respecta a las concepciones acerca de la capacidad de aprendizaje de los estudiantes, tal como con anterioridad había sido observado en el estudio del contexto escolar (Martín, et al. 2014). Ahora bien, que la fuerza de la relación haya sido baja, nos lleva a atender a los distintos factores involucrados. En este sentido, aunque los docentes sostengan la convicción que las capacidades y logros de aprendizaje de los alumnos/as dependen del modo en que se organiza el proceso educativo, lograr traducir esta convicción a la enseñanza implica que se conjuguen múltiples factores, entre los cuales están las características de los docentes (por ejemplo, su formación en estrategias de enseñanza), las características de los estudiantes (por ejemplo, su capacidad de autorregulación del aprendizaje), las condiciones del aula (por ejemplo, el número de alumnos/as en la sala) o de la institución educativa (por ejemplo, el tiempo otorgado para preparar la clase).

Los sucesivos cambios en la educación universitaria nos llevan a valorar las herramientas que aquí hemos presentado para aproximarnos a las cogniciones y conductas de un número amplio de docentes, con la finalidad de generar procesos de reflexión y mejora colectiva. Al mismo tiempo, el estudio posterior de las dimensiones y factores que hemos identificado y de su relación con otras estrategias docentes 0 condiciones del entorno educativo, contribuirá a ampliar y clarificar la relación entre las distintos elementos que componen los procesos de transformación que requieren las instituciones universitarias chilenas para aumentar la equidad de la educación superior.

\section{CONCLUSIONES}

De los resultados expuestos, su análisis y las discusiones que hemos presentados, hemos obtenido las conclusiones que describimos a continuación. Los instrumentos analizados mostraron ser útiles para la comprensión de las cogniciones, valores y prácticas de los docentes universitarios, lo que evidencia su relevancia para conducir procesos de reflexión colectiva en las instituciones educativas. Al respecto vimos: (1) el CDIE es una herramienta válida y fiable en el contexto chileno para acercarse al estudio de las teorías implícitas de los docentes universitarios acerca de la inclusión educativa, al menos en lo que respecta a las concepciones acerca de la capacidad de aprendizaje de los estudiantes; y (2) la EPEI mostró ser un instrumento válido y fiable para describir las prácticas de enseñanza inclusiva de los docentes universitarios; la que puede ser descrita a partir de 3 tres factores: estrategias de enseñanza que promueven un aprendizaje significativo, estrategias de enseñanza que favorecen la co-agencia y estrategias de apoyo a los estudiantes para atender a la diversidad.

En cuanto a los resultados mostrados por el grupo de docentes encuestados concluimos que en general los profesores/as evidencian concepciones y prácticas que promueven la inclusión educativa en la universidad, aun cuando existe dispersión en los avances realizados por la población consultada. En cuanto a las teorías implícitas encontramos que: (1) las concepciones docentes acerca de la capacidad de aprendizaje de los estudiantes tienden levemente hacia un polo que destaca la posibilidad de transformación de las mismas; y (2) en el principio ético de la responsabilidad docente con el aprendizaje de los estudiantes las visiones de los docentes se ubican tanto hacia un polo de baja responsabilidad como hacia un polo de alta responsabilidad, sin predominio de ninguno de estos. Respecto a las prácticas desarrolladas encontramos que (3) los docentes indican emplear con frecuencia estrategias de enseñanza que promueven la inclusión educativa, especialmente en lo que concierne a la enseñanza que fomenta un aprendizaje significativo y al apoyo a los estudiantes para atender a su diversidad. 
En cuanto a la relación entre las variables de interés corroboramos la vinculación entre el pensamiento docente y las acciones desarrolladas en el aula. Al respecto encontramos que: (1) las concepciones acerca de la capacidad de aprendizaje de los estudiantes están relacionadas de manera significativa con las prácticas de enseñanza que promueven la inclusión educativa en sus tres factores; y (2) los factores de prácticas de enseñanza inclusiva identificados mostraron estar significativamente vinculados entre sí. Sin embargo, también observamos (2) que el principio ético de responsabilidad con el aprendizaje de los alumnos/as no se encontró relacionado con las concepciones acerca de la capacidad de aprendizaje, ni se vinculó con las prácticas de enseñanza inclusiva, lo que pone de relieve la necesidad de profundizar en la comprensión de este principio en futuras investigaciones.

\section{AGRADECIMIENTOS}

La presente investigación ha sido desarrollada gracias al esfuerzo coordinado de diversos profesionales pertenecientes a la universidades participantes. Nos interesa agradecer especialmente a los docentes que se motivaron a participar en el estudio, a las autoridades y personal administrativo que nos facilitó el proceso de recolección de datos, y a Javiera Luna Saldias ayudante de esta investigación, sin la cual no hubiésemos logrado acceder a los docentes.

\section{REFERENCIAS}

Abello, R.; Díaz, A.; Pérez, M.; Almeida, L.; Lagos, I.; González, J. y Strickland B., Vivencias e implicación académica en estudiantes universitarios: adaptación y validación de escalas para su evaluación, Estudios Pedagógicos: XXXVIII(2), 7-19 (2012)

Amaro, M.; Méndez, J. y Mendoza, F., Un estudio de las características profesionales del docente universitario para atender a la diversidad, Revista Latinoamericana de Educación Inclusiva: 8(2), 199-216 (2014-2015)

Barkley, E.; Cross, K. y Major, C., Técnicas de aprendizaje colaborativo: manual para el profesorado universitario, 17-31, Morata, Madrid, España (2007)

Booth, T. y Ainscow, M., Guía para la Educación Inclusiva: desarrollando el aprendizaje y la participación en los centros escolares, 21-52, Madrid, OEI-FUHEM, (2015)

Coll, C., Significado y sentido en el aprendizaje escolar. Reflexiones en torno al concepto de aprendizaje significativo, Infancia y Aprendizaje: 11(41), 131-142 (1988)

Creswell, J., Research design: qualitative, quantitative, and mixed methods approaches, 4ª edición, 95-203, California, USA, Sage publications (2013)

Curtis, E. y otros nueve autores, What helps and hinders indigenous student success in higher education health programmes: a qualitative study using the Critical Incident Technique, Higher Education Research \& Development: 34(3), 486-500 (2015)

Devlin, M. y O'Shea, H., Effective university teaching: views of Australian university students from low socioeconomic status backgrounds, Teaching in Higher Education: 17(4), 385-397 (2012)

Diamond, J.; Randolph, A. y Spillane, J., Teachers' expectations and sense of responsibility for student learning: the importance of race, class, and organizational habitus, Anthropology \& Education Quarterly: 35(1), 75-98 (2004)

Echeita, G.; Simón, C.; López, M. y Urbina, C., Educación inclusiva. Sistemas de referencia, coordenadas y vórtices de un proceso dilemático, en Discapacidad e Inclusión. Manual para la docencia, por M. Verdugo y R. Shalock (eds.), pp 329-358, Amarú, Salamanca, España (2013)

Florian, L. y Black-Hawkins, K., Exploring inclusive pedagogy, British Educational Research Journal: 37(5), 813-828 (2011)

García, M.; Sanz, M. y Vilanoba, S., Cuestionario de dilemas para indagar concepciones sobre el aprendizaje en docentes universitarios, Docencia Universitaria: 15, 103-120 (2014)

García, M. y Cotrina, M. La contribución de la universidad al desarrollo de prácticas inclusivas: dilemas y propuestas para avanzar compartiendo, Revista de Educación Inclusiva: 5(1), 123-138 (2012) 
Gavira, R. y Moriña, A., Hidden voices in higher education: inclusive policies and practices in social science and law classrooms, International Journal of Inclusive Education: 19(4), 365-378 (2015)

Gordon, S.; Reid, A. y Petocz, P., Educators' conceptions of student diversity in their classes, Studies in Higher Education: 35(8), 961-974 (2010)

Hart, S.; Drummond, M., y McIntyre, D., Learning without limits: constructing a pedagogy free from determinist beliefs about ability. In The SAGE handbook of special education by L. Florian (ed.), pp 500-516, SAGE Publications, London, UK (2007)

Hockings, C.; Brett, P. y Terentjevs, M., Making a difference. Inclusive learning and teaching in higher education through open educational resources, Distance Education: 33(2), 237-252 (2012)

Hughes, K.; Corcoran, T. y Slee, R., Health-inclusive higher education: listening to students with disabilities or chronic illnesses, Higher Education Research \& Development: en imprenta (2015)

Lauermann, F. y Karabenick, S., The meaning and measure of teachers' sense of responsibility for educational outcomes, Teaching and Teacher Education: 30, 13-26, (2013)

Mansour, N., Consistencies and inconsistencies between science teacher's beliefs and practices, International Journal of Science Education: 35(7), 1230-1275 (2013)

Marchesi, A. y Martín, E., Calidad de la Enseñanza en tiempos de crisis, 83-117, Alianza Editorial, Madrid, España (2014)

Martín, E.; Pozo, J.; Mateos, M.; Martín, A., y Echeverría, M., Infant, primary and secondary teachers' conceptions of learning and teaching and their relation to educational variables, Revista Latinoamericana de Psicología: 46(3), 211-221 (2014)

Mas, O. y Olmos, P. La atención a la diversidad en la educación superior: Una perspectiva desde las competencias docentes, Revista de Educación Inclusiva: 5(1) 159 -174 (2012)

Pisha, B. y Coyne, P., Smart from the start. The promise of universal design for learning, Remedial \& Special Education: 22(4), 197-203 (2001)

Pozo, J., Scheuer, N., Mateos, M. y Pérez, M. Las teorías implícitas sobre el aprendizaje y la enseñanza. En Nuevas formas de pensar la enseñanza y el aprendizaje. Las concepciones de profesores y alumnos, por J. Pozo, N. Scheuer, M. Pérez, M. Mateos, E. Martín y M. De la Cruz (eds.), pp 95-134, Graó, Madrid, España (2011)

Shaw, J., The diversity paradox: does student diversity enhance or challenge excellence?, Journal of Further and Higher Education: 33(4), 321-331 (2009)

Ruiz, R.; Solé, I.; Echeita, G.; Sala, I. y Datsira, M., El principio de Universal Design. Concepto y desarrollos en la enseñanza superior, Revista de Educación: 359, 413-430 (2012)

SIES, Estudios. Servicio de Información de Educación Superior (en la web: http://www.mifuturo.cl/index.php/estudios/estudios-recientes, acceso: 1 de Diciembre 2015), Ministerio de Educación, Chile (2015)

UNESCO, La educación inclusiva: el camino hacia el futuro, Documento de Referencia Conferencia Internacional de Educación, 1-37, Ginebra, Suiza 25 al 28 de Noviembre (2008)

Urbina, C.; Simón, C. y Echeita, G., Concepciones de los profesores acerca de las conductas disruptivas: análisis a partir de un marco inclusivo, Infancia y Aprendizaje: 34(2), 205-217 (2011) 